\title{
Caracterización Morfológica del Componente Tendo-Muscular del Compartimiento Posterior del Muslo
}

\author{
Morphological Characterization of the Tendo-Muscular \\ Component of the Posterior Compartment of the Thigh
}

\author{
Wilson Mauricio Lozano Franco ${ }^{1}$; Pedro Luis Forero Porras ${ }^{2} \&$ Luis Ernesto Ballesteros Acuña ${ }^{3}$
}

LOZANO, W. M.; FORERO, P. P. L. \& BALLESTEROS, A. L. E. Caracterización morfológica del componente tendo-muscular del compartimiento posterior del muslo. Int. J. Morphol., 36(3):829-834, 2018.

RESUMEN: Los aspectos morfológicos del componente tendomuscular del compartimiento posterior han sido descritos en algunos grupos poblacionales. Se evaluó mediante disección directa las características morfológicas y biométricas del compartimento posterior de 50 muslos de especímenes cadavéricos, en una muestra de población colombiana. El $2 \%$ los músculos isquiotibiales se originaron en un tendón común, 58 \% presentó un tendón común para el musculo semitendinoso (MSt) y cabeza larga del músculo bíceps femoral (MBF). El $40 \%$ de la cabeza larga del MBF se originó en el margen lateral de la tuberosidad isquiática (TI) y el MSt en su superficie posterior. En el 98 \% de los casos el músculo semimembranoso (MSm) se originó en el margen medial de la TI y se dispuso como una estructura medial del muslo. No se observaron variaciones en el lugar de inserción de los músculos isquiotibiales. El MSt presentó la mayor longitud (419,5 DE 33,2 mm); el tendón distal con mayor espesor fue el del MBF (8,5 DE 5,6 mm), mientras que el músculo semimembranoso (MSm) presentó el componente miotendinoso más ancho (22,4 DE 6,5 mm). No hubo diferencias morfométricas estadísticamente significativas con relación al lado de presentación. Los hallazgos obtenidos enriquecen los conceptos morfológicos de la región posterior del muslo, aportan a la cualificación de procedimientos de cirugía plástica, ortopédica y al diseño de programas de entrenamiento muscular específico en diferentes disciplinas deportivas.

PALABRAS CLAVE: Músculos isquiotibiales; Músculo semitendinoso; Músculo semimembranoso; Músculo bíceps femoral.

\section{INTRODUCCIÓN}

La región posterior del muslo es ocupada por los músculos semitendinoso, semimembranoso y bíceps femoral, que se extienden entre la cadera y la rodilla, comparten inervación por el nervio isquiático y origen en la tuberosidad isquiática (TI). Esta particularidad hace que estos músculos sean bi-articulares y contribuyan a la extensión del fémur y flexión de rodilla con excepción de la cabeza corta del bíceps femoral, que solo realiza flexión de la rodilla (Olave \& Braga, 2003; Collipal \& Silva, 2011).

El músculo semitendinoso (MSt) presenta su inserción proximal en la TI por un vientre fusiforme pequeño que se continúa con un tendón que ocupa dos tercios del muslo y se inserta en la cara medial de la parte superior de la tibia. Recibe su inervación por la división tibial del nervio isquiático (Olave \& Braga; Moore et al, 2011). De igual manera, el músculo semimembranoso (MSm), se origina a partir de la superficie medial de la TI, de una amplia área ubicada detrás del origen del músculo cuadrado femoral, de la que surgen dos componentes, un tendón de trayectoria vertical que se une progresivamente al vientre muscular y una banda fibrosa que se fusiona a la superficie lateral del tendón de origen; al nivel del tercio medio del muslo se continúa con un vientre muscular, que se inserta en la cara posterior del cóndilo tibial medial; este músculo también recibe inervación de la división tibial del nervio isquiático (Olave, 2005; Moore et al.; Sato et al., 2012).

${ }^{1}$ Universidad Industrial de Santander, Facultad de Salud, Departamento Ciencias Básicas. Bucaramanga, Colombia.

${ }^{2}$ Universidad Industrial de Santander, Facultad de Salud, Departamento de Patología. Bucaramanga, Colombia. Instituto de Medicina Legal y Ciencias Forenses. Bucaramanga, Colombia.

${ }^{3}$ Universidad Industrial de Santander, Facultad de Salud, Departamento Ciencias Básicas. Bucaramanga, Colombia. 
Desde su origen en la TI, la cabeza larga del musculo bíceps femoral (MBF) de aspecto fusiforme, se ubica en la posición lateral del compartimiento posterior del muslo hasta transformarse en un tendón al nivel de la parte inferior de este segmento; allí se une a la cabeza corta, originada en la línea áspera y línea supracondilea lateral del fémur, para cursar por la parte postero-lateral de la rodilla e insertarse en la superficie lateral de la cabeza de la fíbula. La inervación de la cabeza corta está dada por la división fibular común del nervio isquiático; mientras que la cabeza larga es inervada por la por la rama tibial del nervio isquiático (Lippert, 2006; Moore et al.).

Sato et al. describe que la TI tiene dos regiones bien definidas: una antero medial ocupada por el MSm y otra postero lateral para la cabeza larga del MBF y el MSt, quienes se encuentran estrechamente unidos, pero claramente bien diferenciados. La porción tendinosa de la cabeza larga del MBF es gruesa, mientras que el MSt contiene un tendón delgado y corto; ambos tendones están contiguos a las fibras del ligamento sacrotuberoso (Drake et al., 2010; Moore et al.).

A pesar de la fuerte relación entre los tendones de la cabeza larga del MBF y el MSt, las fibras del MBF son más largas y tienen un mayor ángulo de inserción proximal que las del MST, con la implicación funcional de que, al tener mayor cantidad de fibras, puede generar mayor fuerza y potencia en la contracción muscular (Kellis et al., 2010).

En la práctica deportiva la lesión de los componentes tendomusculares del compartimiento posterior del muslo, ha sido reportada como la lesión de no contacto más prevalente en disciplinas como el futbol americano, rugby, futbol y atletismo. Por lo cual, ha sido importante establecer los factores que predisponen a la lesión de los mismos; uno de ellos es la anatomía funcional, conformada por la configuración de la fibra muscular, arquitectura, disposición biauricular y la distribución de la carga entre las inserciones proximales y distales (Opar et al., 2012).

La importancia del conocimiento de la expresión morfológica de los músculos del compartimiento posterior del muslo radica además del concepto académico, en su impacto en el diseño de propuestas de protocolos de ejercicio y entrenamiento para estos grupos musculares específicos, para el manejo y la prevención de sus lesiones, así como para mejorar la eficacia de la contracción muscular en atletas de alto rendimiento (Nacleiro \& Goss-Sampson, 2013). La determinación de las características de los músculos posteriores del muslo ha sido descrita mediante técnicas de disección en otros grupos poblacionales (Miller et al., 2007; Kellis et al.; Fraser et al., 2013). La ausencia de este tipo de información en población mestiza colombiana le da pertinencia a la realización del presente estudio, desarrollado en material cadavérico fresco, con el propósito de obtener información propia de referencia.

\section{MATERIAL Y MÉTODO}

En este estudio descriptivo observacional se evaluaron 50 miembros inferiores provenientes de especímenes cadavéricos no reclamados del Instituto de Medicina Legal de Bucaramanga, Colombia. Se excluyeron los miembros inferiores de sujetos menores de 20 años, con presencia de traumas recientes o cicatrízales, amputaciones supracondileas y deformidades anatómicas.

Con el cadáver en decúbito prono se realizó una incisión mediana posterior comprendida desde el margen lateral de la cresta ilíaca hasta el tercio superior de la pierna, que comprometió piel, tejido celular subcutáneo, fascias femoral y sural (Tank, 2013). Posteriormente, se liberaron los colgajos fascio-cutáneos hacia lateral y medial, lo cual permitió la exposición del primer plano muscular del muslo y pierna; se hizo disección de cada uno de los músculos del compartimiento posterior del muslo y se registraron sus características anatómicas y biométricas tanto de los vientres musculares como de sus tendones. Las variables morfométricas se evaluaron con calibrador digital (Mitutoyo $\left.{ }^{\circledR}\right)$. Igualmente se registraron las variantes tendomusculares con sus características cualitativas y cuantitativas. De cada una de las piezas evaluadas se obtuvieron registros fotográficos digitales.

La base de datos se digitó en Excel y los análisis estadísticos se realizaron en STATA 12.0. Las variables continuas se describieron con sus promedios y desviación estándar, las variables nominales con sus proporciones. Las pruebas estadísticas incluyeron chi cuadrado (c2) y Prueba t de Student. Se consideró significativo un valor de $\mathrm{p}<0,05$.

\section{RESULTADOS}

El $2 \%$ de la muestra, presentó un tendón común para los músculos isquiotibiales, mientras que en el $58 \%$ se observó un tendón común para el MSt y cabeza larga del MBF originados en la superficie central y margen lateral de la TI; así mismo, este par de músculos compartieron vientre muscular en una trayectoria de 104,3 DE 22,9 mm (Fig. 1). En 20 especímenes (40\%) la cabeza larga del MBF se originó en el margen postero-lateral de la TI y el MSt en su su- 


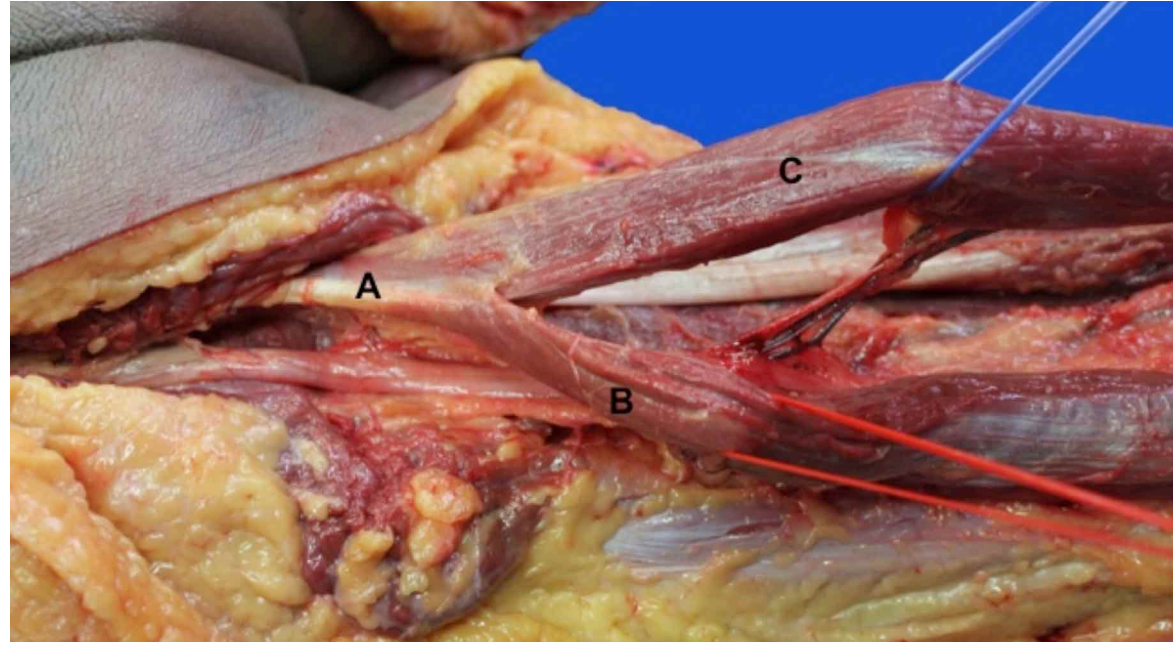

Fig 1. Origen y trayectoria compartida de la cabeza larga del bíceps femoral y del musculo semitendinoso. A. Tendón proximal de la cabeza larga del bíceps femoral y trayectoria compartida del vientre muscular del semitendinoso; B. Vientre muscular de la cabeza larga del bíceps femoral; C. Vientre muscular semitendinoso.

perficie posterior. No hubo diferencias estadísticamente significativas de los orígenes del MSt y de la cabeza larga del MBF con relación al lado de presentación ( $\mathrm{P}=0,23)$.

El MSm, se originó en el margen medial de la TI en 49 casos $(98 \%)$ y se dispuso como una estructura medial del muslo, compartiendo fascia con el musculo aductor mayor en 23 muestras (46\%); mientras que en 54 \% conservó su disposición postero-medial y compartió fascias musculares con el MSt y cabeza larga del MBF. De otro modo en la totalidad de la muestra, el MSt se insertó en la cara medial de la parte superior de la tibia; mientras que la cara posterior del cóndilo tibial medial sirvió de inserción del MSm y la superficie lateral de la cabeza de la fíbula fue la inserción del bíceps femoral.

El MSm presentó una longitud de 37,2 DE 2,69 cm; correspondiendo el $54.6 \%$ a la banda Tendomuscular, $33 \%$ al vientre muscular y $12,4 \%$ al tendón distal. De la longitud de la banda Tendomuscular, el 45,6 \% correspondió a la banda membranosa y el $54,4 \%$ a la porción tendinosa proximal. El ancho de la banda membranosa fue de 25,7 DE 5,8 mm y su espesor de 1,0 DE 0,6 mm, sin diferencias estadísticamente significativas con relación al lado de presentación. ( $\mathrm{P}=0,877 ; 0,700$ respectivamente) (Tabla I).

El tendón distal del MSt ocupó el 30,1 \% (125,5 DE 28,0 mm) de la longitud total del músculo, mientras que a su tendón proximal correspondió el 7,2 \% (29,8 DE $12,6 \mathrm{~mm}$ ). Su vientre muscular presentó una longitud de 261,5 DE 21,16 mm, que correspondió al 62,8 \% de la dimensión del músculo. Así mismo, la dimensión del ancho del tendón distal fue de 24,6 DE 9,2 mm, con un espesor de 2,6 DE 1,2 mm (Tabla II).

La longitud de la cabeza larga del MBF fue de 408,4 DE 28,6 mm, en tanto que la cabeza corta midió $289,6 \mathrm{DE} 27 \mathrm{~mm}$; estas estructuras se fusionaron al nivel de la unión del tercio medio y distal del fémur para formar luego un tendón de 51,9 DE 16,2 $\mathrm{mm}$ de largo, 10,01 DE 3,3 $\mathrm{mm}$ de ancho y espesor de 3,5 DE 1,1 $\mathrm{mm}$. El punto proximal del vientre muscular de la cabeza larga se situó a 56,6 DE 18,2 mm de la TI, mientras que el correspondiente de la cabeza corta se ubicó a 118,8 DE 8,32 mm (Tablas III y IV).

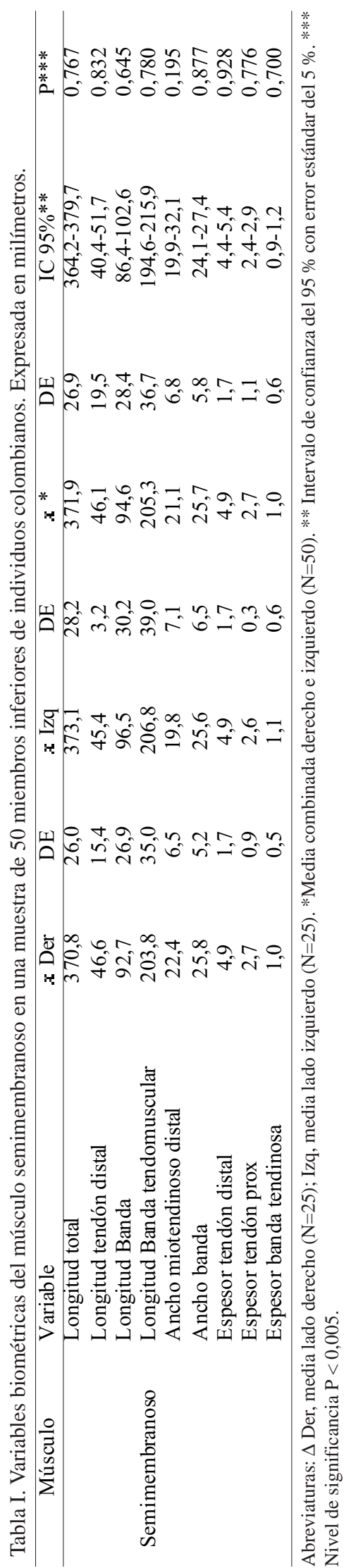


Tabla II. Variables biométricas del músculo semitendinoso en una muestra de 50 miembros inferiores de individuos colombianos. Expresada en milímetros.

\begin{tabular}{|c|c|c|c|c|c|c|c|c|c|}
\hline Músculo & Variable & $x$ Der & $\mathrm{DE}$ & $x \mathrm{Izq}$ & $\mathrm{DE}$ & $x *$ & $\mathrm{DE}$ & IC $95 \% * *$ & $\mathrm{P} * * *$ \\
\hline & Longitud total & 413,3 & 34,6 & 419,5 & 33,2 & 416,4 & 33,7 & $406,5-426,2$ & 0,526 \\
\hline & Longitud tendón corto & 29,1 & 12,3 & 30,6 & 13,2 & 29,8 & 12,6 & $26,2-33,5$ & 0,692 \\
\hline & Longitud Unión Miotendinosa proximal & 88,4 & 26,5 & 85,5 & 29,2 & 86,7 & 27,7 & $78,8-94,3$ & 0,673 \\
\hline & Longitud tendón largo & 128,9 & 31,1 & 122,1 & 24,7 & 125,5 & 28,0 & $117,4-133,6$ & 0,406 \\
\hline \multirow[t]{7}{*}{ Semitendinoso } & Longitud Miotendinosa Distal & 206,0 & 36,1 & 217,3 & 36,0 & 211,6 & 36,1 & $201,2-222,1$ & 0,286 \\
\hline & Ancho tendón largo & 6,2 & 1,5 & 43,0 & 1,3 & 24,6 & 9,2 & $13,3-51,4$ & 0,170 \\
\hline & Ancho miotendinoso proximal & 16,8 & 6,2 & 16,6 & 8,6 & 16,7 & 7,4 & $14,5-18,8$ & 0,932 \\
\hline & Ancho tendón corto & 7,8 & 2,8 & 7,8 & 2,6 & 7,8 & 2,7 & $7,0-8,6$ & 0,948 \\
\hline & Espesor tendón corto & 5,2 & 2,9 & 4,3 & 1,7 & 4,7 & 2,4 & $4,0-5,4$ & 0,185 \\
\hline & Espesor tendomuscular dis tal & 5,0 & 1,4 & 5,3 & 1,6 & 5,7 & 1,5 & $4,7-5,6$ & 0,564 \\
\hline & Espesor tendón largo & 2,4 & 1,2 & 2,8 & 1,2 & 2,6 & 1,2 & $2,3-2,9$ & 0,323 \\
\hline
\end{tabular}

Abreviaturas: Der, media lado derecho $(\mathrm{N}=25)$; Izq, media lado izquierdo $(\mathrm{N}=25)$. *Media combinada derecho e izquierdo $(\mathrm{N}=50)$. ** Intervalo de confianza del $95 \%$ con error estándar del $5 \%$. *** Nivel de significancia $\mathrm{P}<0,005$.

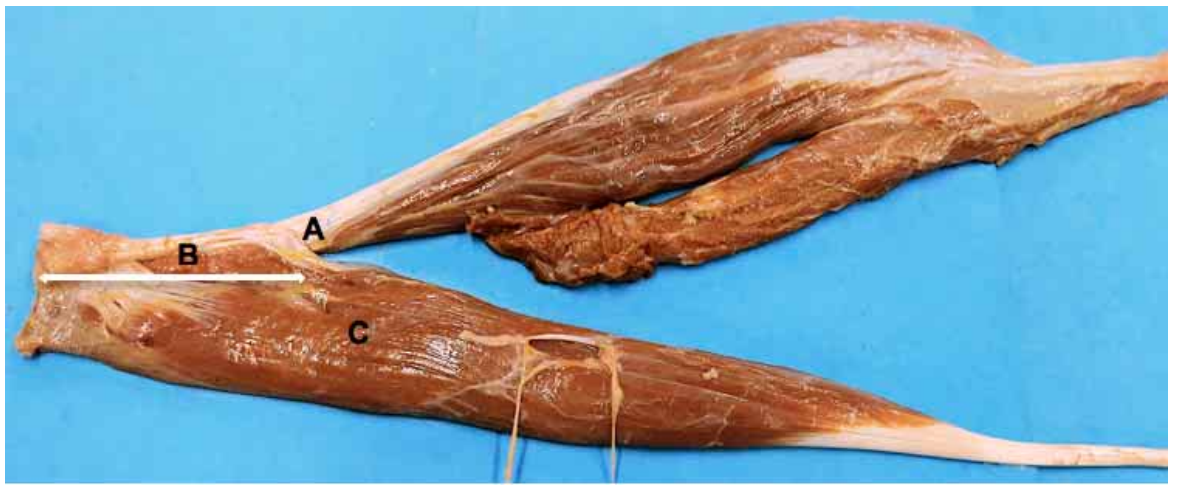

Fig 2. Vista del componente muscular desinsertado; se observa la Trayectoria compartida del tendón proximal de la cabeza larga del bíceps femoral y el vientre muscular del semitendinoso. A. Tendón proximal de la cabeza larga del bíceps femoral; B. Trayectoria compartida del vientre muscular del semitendinoso con el tendón proximal de la cabeza larga del bíceps femoral; C. Vientre muscular semitendinoso.

\section{DISCUSIÓN}

En el presente estudio se observó tres formas de origen de los músculos del compartimiento posterior del fémur; de las cuales, la que presentó una mayor frecuencia (48\%) fue el origen compartido entre la cabeza larga del MBF y el MSt, quienes además comparten trayectoria de su vientre muscular (Fig. 2); rasgo que es concordante con lo reportado en varios estudios previos (Sallay et al., 1996; Miller et al.; Kellis et al.; Sato et al.). Es de resaltar que el origen inusual del semitendinoso reportado por Fraser et al., lo describe con una unión por medio de una cintilla muscular que se proyecta medial y paralela al vientre muscular, separada 7,9 $\mathrm{mm}$ del vientre principal en su inserción proximal en la cara medial de la TI.
Woodley \& Mercer (2005), reportan que la cabeza larga del MBF y el MSt comparten origen, tendón $\mathrm{y}$ vientre muscular con una trayectoria que abarca el tercio proximal del muslo, mientras que en el presente estudio este vientre muscular compartido ocupa el cuarto proximal $(25,2 \%)$ del muslo. Nuestros hallazgos con relación al origen del MSm en el margen medial de la TI coincide con lo reportado en la literatura (Drake et al., 2010; Sato et al.); este origen puede tomar dos trayectorias; en nuestro estudio encontramos una con mayor frecuencia $(78,5 \%)$ en sentido vertical posterior y la segunda más posteromedial $(21,4 \%)$. Así mismo, Miller et al., describen una inserción proximal mas medial con respecto al músculo semimembranoso.

Reina et al. (2013) describe la inserción del MSt mediante tres bandas tendinosas con un promedio de las bandas en la inserción de 40 DE 24 mm ( $\mathrm{p}=0,63$ ); en contraste con el presente estudio donde se observó que el ancho del tendón en la inserción fue de 24,6 DE 9,2 mm ( $\mathrm{p}=0,170)$; esta discrepancia se puede explicar teniendo como referencia el patrón morfológico de inserción predominante en el presente estudio; el cual se caracteriza por la no división del tendón al nivel de la inserción.

La inserción distal del MSm reportada en la cara posterior del cóndilo tibial medial (Olave; Collipal \& Silva), es concordante con lo observado en la totalidad de especímenes del presente trabajo. Benninger \& Delamarter (2013) sugie- 


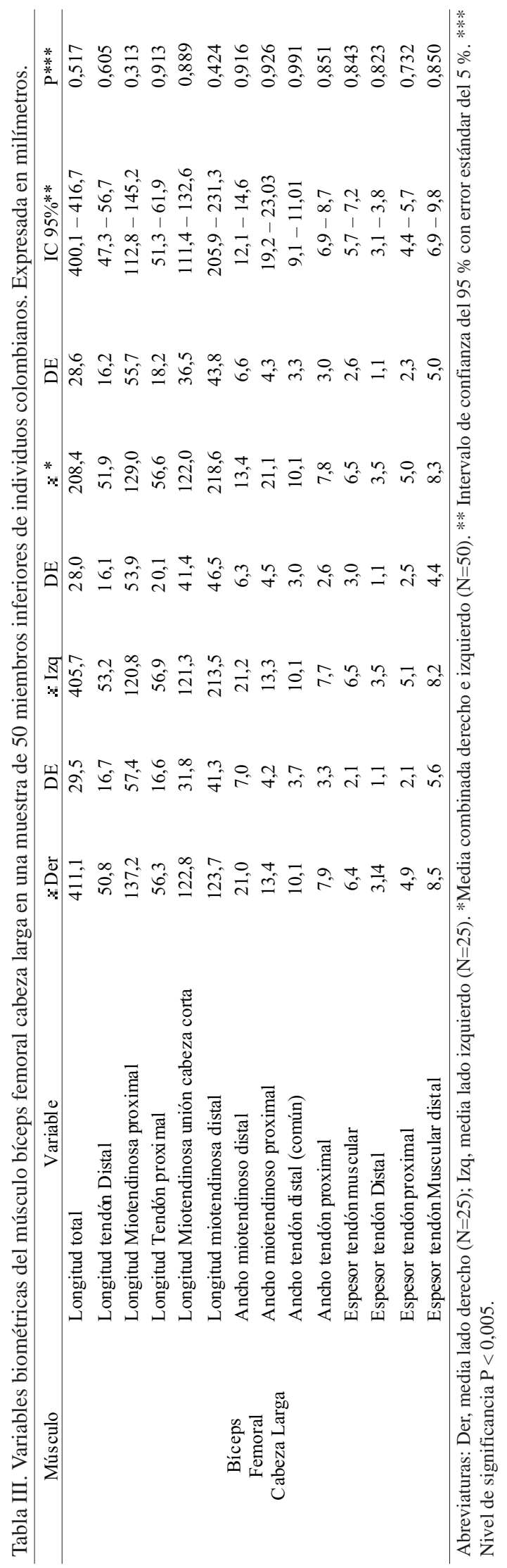

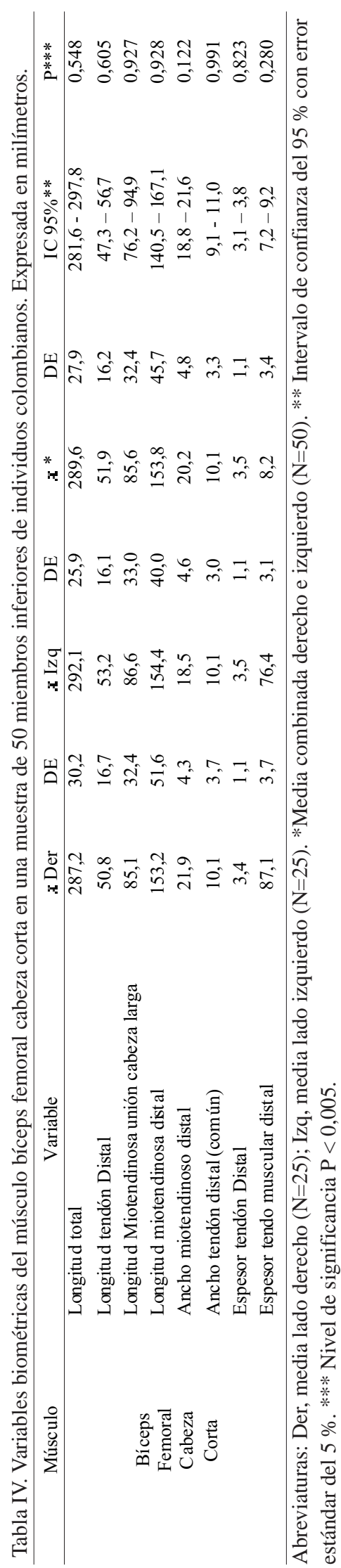

ren que se debe incluir como variación la inserción adicional en el ligamento poplíteo y tendón poplíteo oblicuo.

Para el MBF, Solomon \& Stevenson (2008), reportan inserción al nivel de la superficie lateral y proximal de la tibia y en la parte posterior del tracto iliotibial; característica anatómica que está en desacuerdo con los hallazgos del presente estudio que revelan la inserción del MBF en la totalidad de los especímenes evaluados, en la cabeza de la fíbula, manteniendo el tendón una ubicación postero-lateral con relación al tracto iliotibial sin evidenciar variación en la inserción del MBF.

Los hallazgos biométricos de la longitud global de los músculos del compartimiento posterior del muslo registrados en el presente trabajo (cabeza larga del MBF $438 \mathrm{~mm}$; cabeza corta del MBF bíceps femoral 290 $\mathrm{mm}$ ), son concordantes con lo reportado por Woodley \& Mercer. Este estudio registra de manera detallada dimensiones de los vientres musculares, de los tendones proximales y distales, información no es reportada en estudios previos (Garrett \& Best, 2000).

Las inserciones distales de los músculos de la región posterior del muslo observadas en el presente estudio es concordante con lo reportado en la literatura; mientras que se presenta algún grado de variabilidad en el origen de la cabeza larga del MBF y el MSt. El presente trabajo enriquece la información en torno a las características biométricas y morfo-lógicas de las estructuras muscu- 
lares de la región femoral posterior, usualmente utilizada en los procedimientos de cirugía plástica y ortopédica, para la reconstrucción de componentes miotendionosos y reparaciones ligamentarias.

Además, este conocimiento es útil para diseño de programas de entrenamiento específico en diferentes disciplinas deportivas y en los protocolos de rehabilitación muscular de las estructuras de la región posterior del muslo. Hennessey \& Watson (1993) realizan la exploración clínica de la flexibilidad de la musculatura del compartimiento posterior del muslo en sujetos con historia clínica de lesión isquitiobial versus sujetos sin historial de lesiones, encontrando que no hay cambios significativos en los test clínicos realizados para esta musculatura en particular; no obstante en los atletas y jugadores de futbol se evidencia que en deportistas con antecedente de lesión hay diferencias clínicas en la capacidad de contracción y rango de movimiento activo del muslo con el antecedente de lesión; lo cual, puede ser explicado por el desbalancee muscular producto de un entrenamiento asimétrico sin tener en cuenta la postura, la distribución de la carga de esta musculatura y la variabilidad morfológica de los músculos del compartimiento posterior del muslo.

AGRADECIMIENTOS. Al Instituto de Medicina Legal y Ciencias Forenses de Bucaramanga-Colombia por permitir la disección y registro de los especímenes cadavéricos no reclamados, requeridos en el presente estudio.

LOZANO, W. M.; FORERO, P. P. L. \& BALLESTEROS, A. L. E. Morphological characterization of the tendo-muscular component of the posterior compartment of the thigh. Int. J. Morphol., 36(3):829834,2018

SUMMARY: The morphological aspects of tendon and muscle component of the posterior compartment have been described in some population groups. The morphological and biometric characteristics of the posterior compartment of 50 thighs of cadaverous specimens were evaluated by means of a direct design, in a sample of the Colombian population. The $2 \%$ of the hamstring beats originated in a common tendon, $58 \%$ with a common tendon for the Semitendinous muscle (MSt) and long head of the femoral Biceps muscle (MBF). The $40 \%$ of the long head of the MBF were originated on the lateral margin of the Ischial Tuberosity (TI) and the MSt on its posterior surface. In $98 \%$ of the cases the semimembranous muscle (MSm) originated in the middle margin of the IT and dissolved as a medial structure of the thigh. No variation was observed in the place of the hamstring insertion. The longest time (419.5 DE $33.2 \mathrm{~mm}$ ) the distal tendon with greater thickness was that of the MBF (8.5 OF 5.6 $\mathrm{mm}$ ), while the semimembranosus muscle (MSm) presented the broadest myotendinous component (22.4 SD $6.5 \mathrm{~mm}$ ). There weren't statistically significant morphometric differences in relation to the presentation side. The findings obtained enrich the morphological concepts of the posterior thigh region, contribute to the qualification of plastic surgery, orthopedic procedures and the design of muscle training programs in different sports disciplines.

KEY WORDS: Hamstring muscles; Semitendinosus muscle, Semimembranosus muscle; Femoral biceps muscle.

\section{REFERENCIAS BIBLIOGRÁFICAS}

Collipal, L. E. \& Silva, M. H. Study of anatomy in cadavers and anatomical models. impression of students. Int. J. Morphol., 29(4):1181-5, 2011.

Drake, R. L.; Vogl, W. \& Mitchell, A. W. M. Gray`s Anatomy for Students. $2^{\text {nd }}$ ed. Philadelphia, Churchill Livingstone/Elsevier, 2010.

Fraser, P. R.; Wood, A. R. \& Rosales, A. A. Anatomical variation of the semitendinosus muscle origin. Int. J. Anat. Var., 6:225-7, 2013.

Garrett, W. E. \& Best, T. M. Anatomy, Physiology, and Mechanics of Skeletal Muscle. In: Simon, S. R. (Ed.). Orthopaedic Basic Science. $2^{\text {nd }}$ ed. Park Ridge, American Academy of Orthopedic Surgeons, 2000. pp.704-5.

Hennessey, L. \& Watson, A. W. Flexibility and posture assessment in relation to hamstring injury. Br. J. Sports Med., 27(4):243-6, 1993.

Kellis, E.; Galanis, N.; Natsis, K. \& Kapetanos, G. Muscle architecture variations along the human semitendinosus and biceps femoris (long head) length. $J$. Electromyogr. Kinesiol., 20(6):1237-43, 2010.

Miller, S. L.; Gill, J. \& Webb, G. R. The proximal origin of the hamstrings and surrounding anatomy encountered during repair. A cadaveric study. J. Bone Joint Surg. Am., 89(1):44-8, 2007.

Moore, K. L.; Agur, A. M. R. \& Dalley, A. F. Essential Clinical Anatomy. $4^{\text {th }}$ ed. Baltimore MD, Lippincott Williams \& Wilkins, 2011.

Nacleiro, F. \& Goss-Sampson, M. The effectiveness of different exercises protocols to prevent the incidence of hamstring injury in athletes. OA Sports Med., 1:12-21, 2013.

Olave, E. \& Braga, M. T. T. Innervation of the semitendinous muscle, biometry of the branches and location of its motor points. Int. J. Morphol., 21(2):161$5,2003$.

Olave, E. Innervation of the semimembronosus muscle. Int. J. Morphol., 23(4):363-8, 2005.

Opar, D. A.; Williams, M. D. \& Shield, A. J. Hamstring strain injuries: factors that lead to injury and re-injury. Sports Med., 42(3):209-26, 2012.

Reina, N.; Abbo, O.; Gomez-Brouchet, A.; Chiron, P.; Moscovici, J. \& Laffosse, J. M. Anatomy of the bands of the hamstring tendon: how can we improve harvest quality? Knee, 20(2):90-5, 2013.

Sallay, P. I.; Friedman, R. L.; Coogan, P. G. \& Garrett, W. E. Hamstring muscle injuries among water skiers. Functional outcome and preve;Dion. Am. J. Sports. Med., 24(2):130-6, 1996.

Sato, K.; Nimura, A.; Yamaguchi, K. \& Akita, K. Anatomical study of the proximal origin of hamstring muscles. J. Orthop. Sci., 17(5):614-8, 2012.

Tank, P. W. Grant's Dissector. $15^{\text {th }}$ ed. Philadelphia, Wolter Kluwer Health/ Lippincott Williams \& Wilkins, 2013.

Woodley, S. J. \& Mercer, S. R. Hamstring muscles: architecture and innervation. Cells Tissues Organs, 179(3):125-41, 2005.

Dirección para correspondencia

Wilson Mauricio Lozano Franco

Universidad Industrial de Santander

Facultad de Salud

Departamento Ciencias Básicas

Bucaramanga

COLOMBIA

Email: wilsonmauriciolozano@gmail.com

Recibido : 04-01-2018

Aceptado: 17-02-2018 